\title{
Estudio neuropsicológico de la funcionalidad visual, las estrategias de aprendizaje y la ansiedad en el rendimiento académico
}

\author{
Isabel Martínez-Álvarez(*) y Alicia Lajo Muñoz(**) \\ ${ }^{(*)}$ Universidad a Distancia de Madrid (UDIMA) y $\left.{ }^{(*}\right)$ Universidad de Salamanca
}

\begin{abstract}
RESUMEN
En el contexto educativo actual, los aspectos neurosicológicos cobran gran importancia dada su repercusión en el rendimiento académico. Partiendo de esta premisa, el objetivo de esta investigación es el estudio de la relación entre los movimientos oculares sacádicos, las estrategias de aprendizaje y el nivel de ansiedad, así como su influencia sobre el rendimiento en Lengua. La muestra estaba formada por 69 alumnos de 12-15 años, agrupados en función de su rendimiento. Se trata de un estudio no experimental, descriptivo, de tipo correlacional y comparación de grupos. Por un lado, se ha encontrado una relación inversa entre la lentitud en la lectura (prueba de movimientos sacádicos de King-Devick (1976)) y las estrategias de apoyo al aprendizaje medidas con la Escala ACRA (Román y Gallego, 2008) y entre las estrategias de apoyo y el nivel de ansiedad-estado, medida con el STAIC (Spielberger, Gorsuch y Lushene, 1970). Por otro lado, los alumnos con un rendimiento elevado presentan niveles inferiores de ansiedad-rasgo. Por todo ello, este estudio pone de manifiesto la importancia de intervenciones, que integren la perspectiva neuropsicológica y educativa, centradas en la mejora de las estrategias de aprendizaje y la funcionalidad visual con el fin de promover una disminución de la ansiedad y una mejora en el rendimiento académico del alumnado.
\end{abstract}

Palabras Clave: movimientos oculares, estrategias de aprendizaje, ansiedad, intervención, educación.

\section{Neuropsychological study of visual functionality, learning strategies and anxiety in academic performance}

\section{ABSTRACT}

In the current educational context, neuropsychological aspects are very important given their impact on academic performance. Starting from this premise, the objective of this research is the study of the relationship between saccadic eye movements, learning strategies and the level of anxiety, as well as its influence on language performance. The sample consisted of 69 students aged 12-15 years, grouped according to their performance. It is a non-experimental, descriptive study of correlation type and comparison of groups. On the one hand, an inverse relationship has been found between the slow reading (King-Devick's saccadic movement test (1976)) and the learning support strategies measured with the ACRA Scale (Román and Gallego, 2008) and between the support strategies and the anxiety-state level, measured with the STAIC (Spielberger, Gorsuch y Lushene, 1970). On the other hand, students with high performance have lower anxiety-trait levels. Therefore, this study highlights the importance of interventions, which integrate the neuropsychological and educational perspective, focused on the improvement of learning strategies and visual functionality in order to promote a decrease in anxiety and an improvement in the academic performance of students.

Keywords: ocular movements, learning strategies, anxiety, intervention, education.

\section{Introducción}

En la última década, se ha producido un creciente interés en la aplicación de la Neuropsicología al contexto escolar para optimizar el proceso de enseñanza-aprendizaje, mejorar el rendimiento académico y evitar el fracaso escolar. Las aportaciones que la neuropsicología puede realizar a las prácticas educativas abren nuevos horizontes que benefician a toda la comunidad educativa, proporcionando conocimientos y herramientas que, por un lado, pueden optimizar los procesos cognitivos mediante programas de intervención y, por otro lado, mejorar la detección y evaluación de las dificultades que presenten los alumnos (Martín-Lobo, 2015).

El interés del presente estudio se centra básicamente en tres aspectos fundamentales, en primer lugar, en la importancia de prevenir dificultades relativas a la funcionalidad visual que repercutan de manera negativa en el rendimiento de los alumnos, en segundo lugar, en la necesidad de estudiar qué estrategias de aprendizaje utilizan los estudiantes y su implicación en el aprovechamiento de los recursos cognitivos y metacognitivos para 
presentar un rendimiento positivo y, por último, en la influencia que tienen variables emocionales tales como la ansiedad en el uso deficitario de las habilidades cognitivas y metacognitivas y por extensión, su relación con los resultados académicos.

\section{Funcionalidad visual}

La lectura ha sido ampliamente estudiada a lo largo de la historia. Sin embargo, a pesar de ser un campo en auge debido a sus repercusiones educativas, todavía son escasas las investigaciones centradas en el análisis de la relación entre las bases neuropsicológicas de la visión y las habilidades lectoras. Algunos trabajos, como el de Rosselli, Matute y Ardila (2006), han mostrado que el proceso lector, y por ente el rendimiento académico, se relacionan con aspectos neuropsicológicos como las habilidades visuales y auditivas (Gallardo y Gallego, 2003; Zhang, Yan, Kendrick y Yi Li, 2012). Más concretamente, existen múltiples estudios que analizan la implicación que tiene la buena funcionalidad visual en la lectura (Calvo y Meseguer, 2002; Díaz, Gómez, Jiménez y Martínez, 2004; Lorenzo, 2002; López-Higes, Rubio, Villoria y Mayoral, 2001; Martínez, 2015; Medrano, 2011; Paulson, 2005) y que han puesto de manifiesto su repercusión en el aprendizaje de alumnos de diferentes niveles.

En cuanto a la importancia de los movimientos oculares en la funcionalidad visual, indicar que la rotación que permite tomar distintas posiciones en casi todas las direcciones posibles se debe a que los ojos dentro de las órbitas están inmersos en la grasa orbicular. Esta rotación ocurre gracias a los seis músculos extraoculares (Díaz et al., 2004). Sirven, por tanto, para captar y mantener la fijación de la fóvea sobre un objeto ubicado en el espacio. Concretamente, los movimientos sacádicos se han definido como desplazamientos rápidos de los ojos entre dos puntos de fijación (Gila, Villanueva y Cabeza, 2009; Paulson, 2005).

Mayoritariamente estos movimientos se realizan para desplazar la mirada desde un punto a otro situado fuera del campo de visión central. Por tanto, la información espacial y la atención son fundamentales para orientar la sacada, y estas funciones cognitivas se encuentran en el área 7 de Brodmann, ubicada en el córtex parietal superior. Una vez establecida la fijación en el nuevo punto, entran en juego los mecanismos de la visión central en la corteza estriada y las áreas asociativas visuales occipito temporales para realizar el análisis visual de la forma, el color, etc. del objeto (Gila et al., 2009). El colículo superior se ha señalado como el lugar donde se programan y controlan los movimientos sacádicos. Desde esta estructura parten proyecciones hacia los campos oculares frontales y hacia la corteza parietal superior. Esta región y el área V5/MT, también proyectan hacia la región frontal (Lorenzo, 2002). La programación motora de los movimientos sacádicos tiene lugar en tres áreas frontales: el campo ocular frontal en el área 8, el campo ocular suplementario y la corteza prefrontal dorsolateral.

En la actualidad, la preocupación de conocer cómo influye la correcta funcionalidad de los movimientos sacádicos en aprendizajes básicos como la lectura ha generado un gran número de investigaciones interesadas en estudiar su relación con las dificultades de aprendizaje, así como determinar su implicación en el rendimiento académico de alumnos de Secundaria (Lacámara, 2016; Rubin y Feely, 2009). No son tan numerosas ni rigurosas las investigaciones que estudian la implicación de estos tipos de movimientos y el uso de estrategias de aprendizaje y las halladas demuestran conclusiones e interpretaciones limitadas. En el estudio de Zhang y colaboradores (2012) se vio que las sacadas no solo favorecían la continuidad en la lectura, sino que afectaban a la comprensión, de ahí su impacto en las estrategias de aprendizaje.
Como hemos señalado, la mayor implicación de los estudios con fines educativos en los que analizan los movimientos sacádicos, se han determinado en la lectura, tanto en la velocidad y continuidad, como en la comprensión. Así podemos decir que consisten en pequeños sacádicos progresivos hacia la derecha, cuya duración oscila del 5\% al 20\% del tiempo total de la lectura.

Cuando se ha aprendido a leer, la lectura se transforma en un instrumento imprescindible para el aprendizaje. En la etapa de Educación Secundaria Obligatoria las demandas escolares tienen otras características, tales como que los trabajos de lectura son más largos y por ello es muy importante la precisión y la velocidad para captar los significados con mayor facilidad. Los factores visuales asociados a estas características son la acomodación y la visión binocular, que influyen directamente en la captación de significados de los textos objeto de estudio.

A pesar de que se ha estudiado en profundidad la relación con la lectura, es interesante o empiezan a aparecer estudios centrados en la implicación directa de los movimientos sacádicos en los problemas de aprendizaje. En este ámbito, Metsing y Ferreira (2008) encontraron una relación significativa entre niños con problemas de aprendizaje y alteraciones en los movimientos sacádicos. Entre ellos, se han encontrado fijaciones prolongadas y mayor número de movimientos sacádicos en regresión en niños con problemas de lectura (Medrano, 2011). Por su parte, Vernett, Yang y Kapoula (2011), en su estudio realizaron estimulación transcraneal en jóvenes mientras leían y encontraron un aumento de desconjugación sacádica que ocasionaba desalineación ocular.

Dentro del campo educativo, son muy escasos los programas de intervención neuropsicológica que se han llevado a cabo para la mejora de las habilidades visuales (Ballén, 2016) y es imprescindible ampliar este campo dada la repercusión de estas habilidades en el rendimiento de los estudiantes. En este estudio, nos centramos en el análisis de esta variable esencial y en su relación con el rendimiento para avanzar en este sentido.

\section{Las estrategias de aprendizaje}

Las estrategias de aprendizaje son clave en el campo de la Neuropsicología educativa ya que implican tanto los procesos cognitivos como cuestiones de naturaleza motivacional y metacognitiva que llevan al alumno a planificar, dirigir y controlar su proceso de aprendizaje de diferente manera (Martín-Lobo, Martínez-Álvarez, Muelas, Pradas y Magreñán, 2018). Así pues, se pueden definir las estrategias de aprendizaje como los dispositivos que pone en marcha el estudiante de forma consciente e intencional con el objetivo de alcanzar una meta concreta de aprendizaje. En este proceso tienen un papel especial las condiciones contextuales que rodean a la situación de aprendizaje (Beltrán, Pérez y Ortega, 2006).

Dentro del contexto educativo, Monereo, Castelló, Clariana, Palma, y Pérez (1997) definen las estrategias de aprendizaje como un proceso de toma de decisiones, consciente e intencional, que consiste en seleccionar los conocimientos declarativos, procedimentales y actitudinales necesarios para conseguir un determinado objetivo, siempre de acuerdo con las condiciones de la situación educativa en que se produce la acción.

Por su parte, Román (1993, p. 69) las define como "series eficaces de operaciones mentales que el estudiante utiliza para adquirir, retener y/o recuperar los diferentes tipos de información (conceptos, principios, procedimientos)". Las definiciones consultadas coinciden en que son mecanismos para una gestión eficaz de la información (Roces, González-Pienda y Álvarez, 2002).

Al margen de lo expuesto, cabe recordar que no son muy numerosos los estudios hallados sobre las bases neuropsicológicas 
de las estrategias de aprendizaje. Sin embargo, es importante reflexionar sobre los sistemas encargados de planificar, controlar y verificar las acciones en el desarrollo de la actividad intelectual, el control emocional y la propia capacidad para establecer estrategias de aprendizaje (Herrera, 2007; Muelas, 2015).

Por una parte, hay algunos aspectos investigados que nos orientan hacia la localización de los procesos neuropsicológicos implicados en el aprendizaje. En este sentido, se han comprobado modificaciones en el tamaño de los contactos sinápticos (engrosamiento) debido a la experiencia y también se ha observado un incremento del peso y grosor de la corteza cerebral cuando hay un mayor número de ramificaciones dendríticas. $\mathrm{Y}$ existe además un incremento mayor de células gliales, encargadas de proporcionar aporte metabólico a neuronas más activas. Es conocida también la implicación de los cambios fisiológicos en el aprendizaje, que alteran la respuesta pre o postsináptica. Por otra parte, los cambios en la estructura de la sinapsis proporcionan mecanismos de retención del aprendizaje (Jiménez y Huidobro, 2003; Mora, 2002).

Anatómicamente, la corteza prefrontal tiene un importante papel en la planificación, control, evaluación, etc., del aprendizaje y, por tanto, se ve implicada en el uso de las estrategias de aprendizaje. Recordamos en este punto que la corteza prefrontal dorsolateral se ve involucrada en la programación de los movimientos sacádicos y en el contexto de las estrategias de aprendizaje tiene una función esencial en el procesamiento de reforzadores así como en la planificación de las conductas dirigidas a obtener dichas metas (Bentosela y Mustaca, 2003), por lo que es evidente la repercusión de las funciones ejecutivas en la eficacia de las estrategias de aprendizaje, considerando componentes como la memoria de trabajo (López, 2011).

Por otro lado, se señala que el uso de las estrategias de aprendizaje y su relación con el rendimiento académico ha sido muy estudiado, sobre todo, desde finales del siglo XX. Sin embargo, la mayoría de investigaciones incluyen una muestra universitaria o postgraduada (Camarero, Martín y Herrero, 2000).

Existen algunos estudios con muestra adolescente, como el de Tejedor, González y García (2008), quienes pusieron de manifiesto la importancia de intervenir en el uso de las estrategias con el fin de optimizar el rendimiento a través del estudio de las estrategias que los estudiantes ponen en marcha al aprender con la aplicación del cuestionario ACRA (Roman y Gallego, 2008). Con este instrumento se valora el uso que los estudiantes hacen de las estrategias cuando aprenden clasificándolas en cuatro escalas (de adquisición, de codificación, de recuperación y de apoyo). Estos investigadores encontraron que las estrategias atencionales de exploración eran aquellas que se relacionaban en mayor medida con el rendimiento y, además, que el uso de estas estrategias sufre un ligero decremento a lo largo de los cursos de la etapa secundaria. Otros autores como Brenlla, Porto y Barca (2008), ponen de manifiesto en sus trabajos el impacto de las estrategias de apoyo sobre el rendimiento académico (Bolívar y

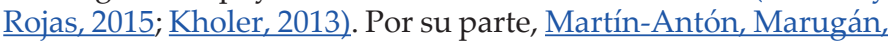
Catalina y Carbonero (2013), señalan que son las estrategias de elaboración las que están más implicadas en el tipo de aprendizaje que se desarrolla en la etapa de Educación Secundaria Obligatoria, ya que las investigaciones reconocen que facilitan el mejor procesamiento de la información y, en consecuencia, su mejor recuperación.

Además, otros estudios hacen referencia a la implicación de las variables contextuales como mediadoras en las estrategias de aprendizaje y finalmente en el rendimiento académico. $\underline{\mathrm{Cid}}$ (2008) indica que la más importante de estas variables es el interés por la tarea o el esfuerzo del estudiante. Por su parte, Cama- rero et al. (2000), encontraron en su estudio que los estudiantes con mayor rendimiento académico utilizan más estrategias metacognitivas, socioafectivas y de control que quienes no. En el presente trabajo se estudiará una de estas variables, como es la ansiedad, por su repercusión en la eficacia del aprendizaje.

En conclusión, numerosas investigaciones se han centrado en el estudio de las estrategias cognitivas y afectivas y de su influencia en el rendimiento pero son muy pocos los trabajos que relacionan las habilidades de pensamiento con los procesos neuropsicológicos del aprendizaje, a pesar de la conveniencia de aplicar los conocimientos que aporta la neuropsicología para mejorar el aprendizaje y aplicarlo en la educación (Béjar, 2014; Cid, 2008; De la Barrera y Donolo, 2009; Martín-Lobo, 2015; Tokuhama-Espinosa, 2008). A través de las neurociencias un educador puede conocer de manera más amplia el cerebro, pudiendo, a partir de esto, mejorar el proceso de enseñanza. De ahí la importancia de estudios como el presente, que se dirijan al análisis profundo de estas variables, así como en su repercusión en el rendimiento académico.

\section{Las repercusiones de la ansiedad}

La ansiedad escolar es definida como un conjunto de síntomas que se agrupan en respuestas cognitivas, psicofisiológicas y motoras emitidas por un individuo ante situaciones escolares que son percibidas como amenazantes y/o peligrosas (García-Fernández, Inglés, Martínez-Monteagudo y Redondo, 2008). Recientes investigaciones coinciden en afirmar que la elevada ansiedad frente a los exámenes está asociada a la baja habilidad para el estudio y al uso de estrategias superficiales de procesamiento de la información, existiendo influencias recíprocas entre dichas variables (Hernández-Pozo, Coronado, Araújo y Cerezo, 2008).

Sin embargo, Contreras et al. (2005), en sus estudios evidenciaron que la autoeficacia está asociada directamente con el rendimiento académico general, mientras que la ansiedad, no. Por lo general, los estudiantes ansiosos se concentran más en la dificultad de la tarea que en el dominio académico, se centran con mayor frecuencia en sus inhibiciones personales, emocionales, así como en los fallos que han tenido en su desempeño previo (Rivas, 1997). En esta misma línea argumental, diversos investigadores han realizado acercamientos a los modelos explicativos de la ansiedad, como, por ejemplo, Eysenck, Santos, Derakshan y Calvo (2007) para quienes la asunción de que la ansiedad aumenta la atención prestada a estímulos relacionados con la amenaza (y a la decisión de cómo responder en las circunstancias que provocan ansiedad) significa que la ansiedad reduce el foco de atención en la tarea corriente.

Por su parte, las estructuras cerebrales generalmente investigadas son el sistema septo-hipocampal (con la amígdala) y los córtex temporal y prefrontal (Garcia et al., 2007). Por ejemplo, estudios estructurales con imágenes de resonancia magnética han revelado correlaciones neurales importantes de los trastornos de ansiedad con el hipocampo, la amígdala y la corteza prefrontal (Hasler, Drevets, Manji, y Charney, 2004).

Asimismo, en la actualidad, se acepta que las emociones son controladas por un circuito nervioso que amplifica y mantiene las reacciones emocionales. Este circuito integra el hipotálamo, desde donde parten las reacciones viscerales (hambre, sed, temperatura, sueño, etc.), la amígdala, que controla la expresión del comportamiento, la corteza cingulada, que produce la conciencia de la emoción, y el hipocampo, que participa en la memoria de las emociones (Castro-Sierra, León, Domínguez, y Rivera, 2007). Específicamente, el eje hipotálamo-pituitaria-adrenal (HPA) ha 
sido implicado en la patogénesis de la ansiedad. La hormona de liberación de corticotrofina (CRH) es el mediador primario de la actividad HPA. El estrés induce la liberación de CRH del hipotálamo, que causa la liberación de adrenocorticotrofina de la glándula pituitaria y por último conduce a la liberación de hormonas glucocorticoides del estrés de la glándula suprarrenal (Miller y O'Callaghan, 2002).

En la literatura científica revisada también es común distinguir entre ansiedad-rasgo y ansiedad-estado. Así las cosas, el modelo estado-rasgo, tal y como se entiende en la actualidad, fue desarrollado inicialmente por Cattell y Scheer (1961). Este modelo distingue dos aspectos fundamentales de la ansiedad: la ansiedad como un estado emocional transitorio y la ansiedad como un estado más permanente, que corresponde a un rasgo de la personalidad.

Por un lado, la ansiedad-estado podemos describirla como sentimientos subjetivos conscientemente percibidos como inadecuados y la tensión acompañada de un aumento de la activación (excitación) en el sistema nervioso autónomo. Por tanto, se conceptualiza como un estado en el que se da un patrón transitorio de emociones creadas por un ambiente estresante, incluyendo síntomas fisiológicos y de aprensión, preocupación y tensión (Clarke, McLeod y Shirazee, 2008). Por otro lado, la ansiedad-rasgo se define como una predisposición en el comportamiento, independiente del tiempo, lo que lleva a una tendencia para percibir una amplia gama de situaciones como peligrosas o amenazantes. Así, la ansiedad-rasgo se refiere a las diferencias individuales relativamente estables en la propensión a la ansiedad (Clarke et al, 2008). En consonancia con lo señalado, las puntuaciones de ansiedad-rasgo son menos sensibles a los cambios debido a situaciones ambientales y se mantienen relativamente constantes a lo largo del tiempo (Hackfort y Schwenkmezger, 1993). Es por ello por lo que la teoría neurofisiológica que explica la ansiedad-rasgo pretende caracterizarla en términos del funcionamiento del sistema nervioso central (Calvo y Cano-Vindel, 1997), defendiendo que la susceptibilidad en relación con la ansiedad depende del aumento de reactividad del sistema de inhibición comportamental, que implica sobre todo al sistema septohipocampal.

Específicamente, autores como Furlán, Sánchez, Heredia, Piemontesi e Illbele (2009) analizaron cuatro dimensiones de ansiedad (preocupación, emocionalidad, interferencia y falta de confianza) y las estrategias de aprendizaje en alumnos universitarios y se halló que los estudiantes con más ansiedad utilizaban estrategias de repetición y búsqueda de ayuda académica y los de baja ansiedad, estrategias de estudio reflexivo, lo cual repercutía en su rendimiento. En su caso, Soto, Da Cuña, Gutiérrez y González (2012) estudiaron las relaciones entre el uso de estrategias de aprendizaje cognitivas y la percepción de los estresores académicos. Entre las conclusiones que destacan, señalamos que encontraron que las estrategias de elaboración o planificación no afectan a la percepción de los estresores académicos. Mientras que la estrategia de memorización actúa como factor de riesgo y la estrategia de selección como un factor atenuante.

Así pues, la evidencia empírica reciente (Delgado, et al., 2018; González Fragoso, Guevara Benítez, Jiménez Rodríguez y Alcázar Olán, 2018), pone de relieve que los niveles de ansiedad escolar se incrementan sustancialmente en estudiantes de E.S.O. y que esto afecta significativamente al rendimiento (Rodríguez y Rosquete, 2018). En esta línea, algunas investigaciones han encontrado que es alrededor de los 11-12 años cuando se encuentran los niveles más elevados, coincidiendo en atribuir este aumento a mayores exigencias educativas (Tejero, 2006).

Por último, y como se ha podido comprobar, a pesar de la relevancia de las habilidades visuales, las estrategias de apren- dizaje y la ansiedad en el contexto de la Neuropsicología y la Educación, son escasas las investigaciones dedicadas al estudio de las relaciones entre estas variables, así como en su impacto sobre el rendimiento. Partiendo de esta carencia, en este trabajo el objetivo general fue llevar a cabo una investigación para analizar los movimientos oculares sacádicos, las estrategias de aprendizaje y la ansiedad que manifiestan los alumnos de $1^{\mathrm{o}}$ E.S.O., la relación entre las variables y comprobar su repercusión en el rendimiento académico de la asignatura de Lengua Castellana.

A partir de este objetivo general, se proponen como objetivos específicos los siguientes:

- Analizar los movimientos sacádicos, las estrategias de aprendizaje que ponen en marcha y el nivel de ansiedad de los participantes de este estudio.

- Estudiar las relaciones entre los movimientos sacádicos, las estrategias de aprendizaje, la ansiedad-estado, la ansiedad-rasgo y el rendimiento académico en la asignatura de Lengua Castellana.

- Comparar ambos grupos (alto y bajo rendimiento) en cuanto a las variables indicadas (movimientos sacádicos, estrategias de aprendizaje, ansiedad-estado y ansiedad-rasgo).

Por otro lado, tomando como referencia los objetivos anteriormente señalados, se han formulado las hipótesis mencionadas a continuación:

- $\mathrm{H}_{1}$ : Se espera hallar niveles adecuados, dentro de los valores promedio, en relación con los movimientos sacádicos al leer, el uso de las estrategias de aprendizaje y el nivel de ansiedad de la muestra.

- $\mathrm{H}_{2}$ : Se espera encontrar una relación significativa entre la eficaz funcionalidad visual, la adecuada puesta en marcha de estrategias de aprendizaje y un bajo nivel de ansiedad.

- $\mathrm{H}_{3}$ : Se espera encontrar que los alumnos con alto rendimiento académico presenten una mayor fluidez lectora, pongan en marcha estrategias de aprendizaje de manera adecuada y presenten niveles bajos de ansiedad en comparación con los alumnos con un bajo rendimiento.

\section{Método}

\section{Diseño}

Se trata de un estudio no experimental, descriptivo, de tipo correlacional y comparación de grupos. La variable independiente era el rendimiento académico, con dos niveles: alto y bajo, siendo las variables dependientes tres: los movimientos sacádicos, las estrategias de aprendizaje y el nivel de ansiedad.

\section{Participantes}

Los sujetos participantes estaban matriculados en el curso $1^{\mathrm{o}}$ E.S.O, en un colegio concertado de nivel sociocultural y económico medio-alto de la ciudad de Valladolid. El centro es de línea dos en Educación Infantil y de línea tres en Educación Primaria y Educación Secundaria Obligatoria. Se realizó un muestreo incidental del que resultó un total de 69 sujetos participantes, 33 mujeres y 36 varones, con edades comprendidas entre los 12 y los 15 años $(M=13,16 ; S D=0,92)$. Todos ellos participaron de manera voluntaria, con autorización de los padres y/o tutores y 
ninguno de ellos presentaba Necesidades Específicas de Apoyo Educativo (NEAE). Los alumnos fueron divididos en dos grupos en base a su rendimiento en Lengua, alumnos con alto rendimiento (37 sujetos; $53,62 \%$ ) y alumnos con bajo rendimiento (32 sujetos; $46,38 \%)$.

\section{Variables e Instrumentos de recogida de información}

La variable independiente del presente estudio es el rendimiento académico en Lengua, mientras que las variables dependientes son: Movimientos oculares sacádicos, estrategias de aprendizaje, ansiedad estado y ansiedad rasgo. Con el fin de evaluar la funcionalidad visual se utilizó la Prueba de King-Devick. Este test valora la función oculomotora en base a la velocidad y número de errores con la que se ven, reconocen y verbalizan una serie de 40 números dispuestos horizontalmente. El test King-Devick fue elaborada por King y Devick (1976) para niños de edades comprendidas entre los 6 y los 15 años. Consta de cuatro tarjetas, una de ellas de demostración y las tres restantes para evaluar la funcionalidad visual a través de la lectura de ocho líneas con cinco números en cada una de ellas. Los números se presentan separados de forma aleatoria para conseguir que los movimientos oculares que hacen los niños sean similares a los que ponen en marcha durante el proceso lector. Se anota tanto el tiempo empleado por el participante en cada tarjeta como el número de errores cometidos para finalmente compararlo con los baremos establecidos por edad. El coeficiente de fiabilidad de la prueba $K D$ es elevado $(\alpha=0,97)$.

La evaluación de las estrategias de aprendizaje se realizó a través de la escala de estrategias de aprendizaje ACRA (Román y Gallego, 2008). Este cuestionario de respuestas cerradas está compuesto por cuatro escalas independientes: Adquisición (siete estrategias), Codificación (trece estrategias), Recuperación (cuatro estrategias) y Apoyo al procesamiento (nueve estrategias). El estudiante debe contestar a cada uno de los ítems que se presentan a través de una escala Likert de cuatro opciones que varían entre "nunca o casi nunca" y "siempre". Para cada una de las escalas se obtiene una puntuación directa que se transforma en base a los baremos de la prueba en puntuación centil. El coeficiente de fiabilidad de la escala ACRA total es alto $(\alpha=0,97)$.

Para la evaluación de la variable ansiedad, se aplicó la adaptación española del "Cuestionario de ansiedad estado-rasgo" (State-Trait Anxiety Inventory, STAI) (Spielberger, et al., 1970). Este instrumento es un autoinforme de 40 ítems que evalúa, por un lado, la ansiedad como estado (transitoria) y, por el otro, la ansiedad como rasgo (estable). Cada subescala de la prueba es independiente y está formada por 20 ítems de respuesta tipo Likert con cuatro niveles que varían desde "casi nunca/nada" hasta "siempre/casi siempre). La puntuación de cada escala puede encontrarse entre 0 y 60 y en muestras españolas, los niveles de consistencia interna se encuentran entre 0,84 y 0,93 .

\section{Procedimiento}

Primeramente, se llevó a cabo una entrevista inicial con la Orientadora de Secundaria y los Jefes de Estudio del centro educativo para exponer los objetivos del estudio y solicitar permiso para la aplicación de los instrumentos mencionados en la muestra descrita. Posteriormente, se presentó al director del centro la solicitud de colaboración por escrito.

Una vez autorizado el estudio, se envió una carta informativa a todas las familias de los alumnos seleccionados para expli- carles en términos generales el estudio y solicitar por escrito el consentimiento informado para autorizar a sus hijos a participar.

Los instrumentos STAIC y ACRA fueron administrados colectivamente en el aula en horas de la mañana, en presencia del tutor del grupo, en 3 sesiones distintas de una duración aproximada de 60 minutos cada una (una sesión por cada clase). El orden de aplicación fue primero el STAIC y posteriormente la ACRA. Los alumnos indicaron sus datos personales y a continuación se leyeron en voz alta las instrucciones, resaltando la importancia de no dejar ninguna pregunta sin contestar. La autora del trabajo estuvo presente durante la administración de las pruebas para aclarar posibles dudas y verificar que se cumplimentaba correctamente por parte de los estudiantes.

La prueba K-D fue administrada por dos evaluadoras (una de las autoras del estudio y la otra orientadora del colegio), durante 3 sesiones (3 horas aproximadamente) distintas en cada uno de los despachos de orientación del colegio, saliendo los alumnos de manera individual de su grupo-clase. Los estímulos del test K-D se han presentado en 4 hojas de papel tamaño A-4 plastificadas, correspondiendo a la carta de demostración, carta I, II y III, registrándose el tiempo y los errores de cada sujeto evaluado.

\section{Análisis de datos}

Para el análisis de datos se ha empleado el Paquete Estadístico SPSS versión 22. El análisis del estudio está basado en la estadística de tipo descriptivo y correlacional y de comparación de grupos. Para ello se han calculado diferentes índices estadísticos tras comprobar previamente los principios de normalidad de las variables de estudio a través de la prueba no paramétrica Kolmogorov-Smirnov: Tiempo en $\operatorname{KD}(Z=0,90 ; p=0,40)$; Errores en $\mathrm{KD}(Z=1,90 ; p=0,30)$; escala de adquisición $(Z=0,75 ; p=0,63)$; escala de codificación $(Z=0,82 ; p=0,51)$; escala de recuperación $(Z=0,48$; $p=0,98)$; escala de apoyo $(Z=0,92 ; p=0,37)$; escala de ansiedad-estado $(Z=1,18 ; p=0,13)$; escala de ansiedad-rasgo $(Z=0,92 ; p=0,37)$. En primer lugar, a nivel descriptivo, se han analizado la media y la desviación típica de las puntuaciones obtenidas en cada una de las variables. En segundo lugar, se halló el Coeficiente de Correlación de Pearson, que mide la relación entre variables. En tercer lugar, para la comparación de los grupos se ha empleado la prueba ANOVA, que nos permite comparar la media de las variables estudiadas en los dos grupos independientes, en este caso, en el grupo de alto y de bajo rendimiento. En este análisis se han incluido como covariables el sexo y la edad de los participantes con el fin de controlar posibles efectos de las mismas sobre los resultados.

\section{Resultados}

\section{Análisis descriptivo}

A continuación, se presentan las medias y desviaciones típicas obtenidas por la muestra en cada uno de los aspectos evaluados.

Como puede verse en la Tabla 1, los alumnos cometieron escasos errores en la prueba K-D y la media del tiempo requerido para completar dicha prueba se encuentra dentro de los valores considerados medios por lo que la muestra del estudio se adecua a los valores estandarizados. En cuanto a las escalas de la prueba ACRA, la media de las puntuaciones en cada una de las escalas, así como a total, se encuentra también en valores promedios. Por último, el nivel de ansiedad-estado es elevado y el nivel de ansiedad-rasgo se encuentra en unos valores medios. 
Tabla 1. Análisis descriptivos.

\begin{tabular}{crc}
\hline Variable & Media & Desviación típica \\
\hline Nota & 5,03 & 1,96 \\
Tiempo K-D & 52,09 & 9,18 \\
Errores K-D & 0,45 & 0,76 \\
ACRA Global & 300,13 & 59,59 \\
Adquisición & 52,87 & 9,69 \\
Codificación & 106,52 & 24,89 \\
Recuperación & 46,83 & 10,35 \\
Apoyo & 93,91 & 21,79 \\
\hline
\end{tabular}

\section{Análisis de correlación}

Por su parte, en la Tabla 2 muestra las correlaciones entre las distintas variables:

Tabla 2. Análisis correlacionales realizados.

\begin{tabular}{|c|c|c|c|c|c|c|c|c|c|c|}
\hline Criterio & (1) & (2) & (3) & (4) & (5) & (6) & (7) & (8) & (9) & (10) \\
\hline (1) Nota & 1 & $-0,04$ & $-0,06$ & $-0,03$ & $-0,02$ & $-0,04$ & $-0,04$ & 0,00 & 0,00 & $-0,35^{* *}$ \\
\hline (2) Tiempo K-D & & 1 & 0,15 & $-0,22$ & $-0,10$ & $-0,18$ & $-0,15$ & $-0,28^{*}$ & $-0,11$ & 0,01 \\
\hline (3) Errores K-D & & & 1 & 0,01 & 0,02 & $-0,01$ & $-0,04$ & 0,05 & $-0,12$ & 0,03 \\
\hline (4) ACRA global & & & & 1 & $0,74^{*}$ & $0,93^{*}$ & $0,90^{*}$ & $0,91^{*}$ & 0,14 & $-0,05$ \\
\hline (5) Adquisición & & & & & 1 & $0,63^{*}$ & $0,57^{*}$ & $0,58^{*}$ & $-0,04$ & $-0,05$ \\
\hline (6) Codificación & & & & & & 1 & $0,81^{*}$ & $0,75^{*}$ & 0,11 & $-0,07$ \\
\hline (7) Recuperación & & & & & & & 1 & $0,81^{*}$ & 0,04 & 0,02 \\
\hline (8) Apoyo & & & & & & & & 1 & $0,26^{*}$ & $-0,05$ \\
\hline (9) Ansiedad Estado & & & & & & & & & 1 & 0,15 \\
\hline (10) Ansiedad Rasgo & & & & & & & & & & 1 \\
\hline
\end{tabular}

${ }^{*} p<0,05 ;{ }^{* *} p<0,01$

Como puede advertirse en la tabla superior, los resultados muestran una correlación significativa negativa entre las estrategias de apoyo al aprendizaje y el tiempo empleado en la prueba K-D, y una correlación significativa positiva entre las estrategias de apoyo y la ansiedad-estado. También se detecta una correlación significativa negativa entre la ansiedad-rasgo y el rendimiento académico en Lengua.

\section{Análisis de comparación entre grupos}

En este caso, en la Tabla 3 se muestran los estadísticos descriptivos del grupo de alto y bajo rendimiento. Según se observa, los grupos de alto y bajo rendimiento obtienen medias similares en todas las variables medidas excepto en el nivel de ansiedad rasgo.

Finalmente, en la Tabla 4 se muestra la comparación de las variables (movimientos oculares sacádicos, estrategias de aprendizaje y ansiedad) entre los grupos de alto y bajo rendimiento.

Tabla 3. Estadísticos descriptivos de las variables en grupos bajo y alto rendimiento.

\begin{tabular}{lcccc}
\hline Criterio & \multicolumn{4}{c}{ Grupo } \\
& \multicolumn{2}{c}{ Bajo rendimiento (N=32) } & Alto rendimiento (N=37) \\
& $\mathrm{M}$ & $\mathrm{DT}$ & $\mathrm{M}$ & $\mathrm{DT}$ \\
\hline Tiempo K-D & 52,47 & 7,44 & 51,76 & 10,54 \\
Errores K-D & 0,50 & 0,80 & 0,41 & 0,72 \\
ACRA (Global) & 301,91 & 48,26 & 298,59 & 68,53 \\
Adquisición & 53,09 & 8,51 & 52,68 & 10,72 \\
Codificación & 107,69 & 21,43 & 105,51 & 27,79 \\
Recuperación & 47,31 & 9,74 & 46,41 & 10,97 \\
Apoyo & 93,81 & 17,9 & 94 & 24,91 \\
AE & 35,5 & 4,75 & 35,49 & 3,83 \\
AR & 38,69 & 8,16 & 32,81 & 7,93 \\
\hline
\end{tabular}


Tabla 4. Comparación de grupos.

\begin{tabular}{lccc}
\hline Criterio & $\boldsymbol{F}$ & $p$ & $\boldsymbol{y}_{\mathrm{p}}{ }^{2}$ \\
\hline Tiempo KD & 0,10 & 0,75 & 0,02 \\
Errores KD & 0,26 & 0,61 & 0,04 \\
ACRA global & 0,11 & 0,75 & 0,02 \\
Adquisición & 0,03 & 0,86 & 0,00 \\
Codificación & 0,13 & 0,72 & 0,02 \\
Recuperación & 0,13 & 0,72 & 0,02 \\
Apoyo & 0,01 & 0,97 & 0,00 \\
AE & 0,01 & 0,98 & 0,00 \\
AR & $\mathbf{9 , 1 7 *}$ & $\mathbf{, 0 0}$ & $\mathbf{0 , 1 2}$ \\
\hline
\end{tabular}

En cuanto a la comparación entre los grupos, se observan diferencias estadísticamente significativas entre el grupo de alto y bajo rendimiento en la variable ansiedad-rasgo con un tamaño del efecto moderado.

\section{Discusión}

El objetivo general de este estudio era analizar la relación entre los movimientos oculares sacádicos, las estrategias de aprendizaje y la ansiedad, así como su influencia sobre el rendimiento académico en Lengua en alumnos de ESO. A partir de los resultados obtenidos se han encontrado ciertas tendencias que resaltan las relaciones entre las variables estudiadas, así como su relevancia en el rendimiento de los alumnos, y que van en la línea de estudios previos ya realizados en este campo (Delgado et al., 2018; Lacámara, 2016; Martín-Lobo et al., 2018).

En cuanto a la primera hipótesis planteada, a nivel descriptivo encontramos que los alumnos de la muestra cometieron escasos errores en la prueba K-D y el tiempo medio de ejecución se encontraba dentro de los valores estandarizados. En cuanto a las escalas de la prueba ACRA, obtuvieron resultados que pueden situarse en el intervalo de la mitad superior de las puntuaciones. Respecto a la ansiedad, mostraron un mayor nivel en la ansiedad-estado que en la ansiedad-rasgo, llegando la primera a percentiles muy elevados. Este último resultado apoya los ya encontrados previamente por autores como González Fragoso y sus colaboradores en 2018 con alumnos de secundaria y pone de relieve la relevancia de atender a factores socioemocionales en los estudiantes de secundaria.

A partir de la segunda hipótesis planteada se esperaba encontrar una relación significativa entre la eficaz funcionalidad visual, la adecuada puesta en marcha de estrategias de aprendizaje y un bajo nivel de ansiedad. A la vista de los resultados, esta suposición se cumple tan solo parcialmente, ya que no se da una correlación significativa entre el tiempo de ejecución de la prueba K-D y las puntuaciones globales de la escala ACRA. Sin embargo, se ha observado una relación negativa entre el tiempo de ejecución de la prueba K-D y una de las escalas que integran la ACRA, la escala de apoyo al proceso de aprendizaje, es decir, los alumnos que ponen en marcha más estas estrategias son los que suelen consumir menos tiempo en la prueba K-D. Adicionalmente, se ha encontrado una correlación significativa negativa, entre la ansiedad-rasgo y el rendimiento académico en Lengua, lo que va en la línea de los trabajos realizados por Rodríguez y Rosquete (2018), que ya encontraron una relación entre el nivel de ansiedad de los estudiantes y su rendimiento académico global. También existe una correlación significativa, en este caso positiva, entre las estrategias de apoyo y la ansiedad-estado. La relación entre ambas variables es coherente, puesto que las estrategias de apoyo integran las estrategias metacognitivas, socio-afectivas y motivacionales (Román y Gallego, 2008).

La tercera hipótesis del estudio también se cumple parcialmente, puesto que no se han observado diferencias significativas respecto a la funcionalidad visual y las estrategias de aprendizaje entre el grupo de alto y bajo rendimiento, hecho que contrasta con investigaciones previas en el campo (Lacámara, 2016; Martín-Lobo et al., 2018). Un posible factor explicativo a este hecho puede estar relacionado con la valoración del rendimiento de la asignatura de lengua, ya que se ha tomado como valor cuantitativo la nota media obtenida, de manera que se podrían obtener otros resultados si este valor incluyese diferentes porcentajes desglosando el rendimiento en cada una de los criterios de evaluación considerados (comprensión lectora, velocidad lectora, expresión escrita, reglas gramaticales, expresión oral, etc.), en los que tiene una incidencia más directa la funcionalidad visual, por ejemplo. En cuanto a las estrategias de aprendizaje, es necesario incidir mayormente en el estudio de su posible repercusión en el rendimiento global de los estudiantes, así como en las diferentes áreas, tal y como encontraron Martín-Lobo y sus colegas (2018) en su reciente estudio.

Como se ha analizado en el párrafo previo, algunos de los resultados de este estudio no estarían en consonancia con la investigación previa sobre la base neuropsicológica del fracaso escolar, donde se pone de manifiesto que los alumnos que suspenden asignaturas tienen un menor nivel de desarrollo en factores visuales, auditivos, motrices, espacio-temporales, de lenguaje y de memoria, con una diferencia significativa a favor de los estudiantes que aprobaban en todas las asignaturas (Martín-Lobo et al., 2018; Santiuste, Martín-Lobo y Ayala, 2006). Por este motivo, resulta esencial continuar avanzando en este campo para poder clarificar en mayor medida las repercusiones de las variables neuropsicológicas en el rendimiento académico.

A diferencia de los resultados analizados hasta ahora en cuanto a la tercera hipótesis, en este estudio sí se han encontrado diferencias significativas entre ambos grupos en la variable ansiedad-rasgo, como ya puso de manifiesto Lacámara (2016). Recordamos que entendemos por ansiedad-rasgo la predisposición en el comportamiento, independiente del tiempo, lo que lleva a una tendencia para percibir una amplia gama de situaciones como peligrosas o amenazantes y por tanto repercute de manera sostenida en el rendimiento. La ansiedad-estado la podemos describir como un estado en el que se da un patrón transitorio 
de emociones creadas por un ambiente estresante, incluyendo síntomas fisiológicos y de aprensión, preocupación y tensión (Clarke et al., 2008). En esta línea, otras investigaciones previas (Contreras y otros, 2005), ya evidenciaron que la autoeficacia está asociada directamente con el rendimiento académico general. Además, los estudiantes ansiosos se concentran más en la dificultad de la tarea que en el dominio académico, se centran con mayor frecuencia en sus inhibiciones personales, emocionales, así como en los fallos que han tenido en su desempeño previo (Rivas, 1997), por lo que es evidente que tienen un mayor nivel de ansiedad mantenido en el tiempo.

En síntesis, los resultados de este estudio ofrecen indicios acerca de la existencia de relaciones entre las estrategias de apoyo y el tiempo empleado en la prueba K-D; entre las estrategias de apoyo y la ansiedad-estado y entre la ansiedad-rasgo y el rendimiento en la asignatura de Lengua. Respecto a la comparación de grupos, podemos decir, aunque con cautela, que los estudiantes que presentan un elevado nivel de ansiedad-rasgo rinden menos que aquellos con bajo nivel, lo cual debe ser tenido en cuenta a la hora de intervenir con los estudiantes en el contexto educativo.

Atendiendo a los resultados de esta investigación y a otros trabajos previos que apuntan a la relación e influencia de estas variables en el rendimiento, podemos corroborar la importancia de la enseñanza explícita de las estrategias neuropsicológicas en el aula ya que son necesarias para aprender a aprender (Muelas, 2015). Ayuda a que las clases sean activas, flexibles y tendientes a lograr aprendizajes significativos, mejora la calidad del proceso de enseñanza-aprendizaje y eleva el rendimiento académico y personal de los alumnos (Northon, 2012).

Además, los datos de esta investigación revelan la importancia de trabajar los aspectos motivacionales y afectivos en los alumnos, que a veces no se trabajan explícitamente debido a la carga curricular de las asignaturas.

La edad de los estudiantes de secundaria implica cambios que a menudo repercuten de manera negativa en los alumnos (mayores exigencias curriculares, cambios organizativos, mayor número de profesores y asignaturas, etc.), lo que desencadena situaciones estresantes que vulneran la estabilidad y el bienestar de los alumnos e influyen en el rendimiento académico. Si a esto añadimos los grandes cambios personales, de desarrollo neuropsicológico, y sociales de la pubertad, tenemos el contexto idóneo para realizar propuestas educativas de prevención que asuman las características neuropsicológicas de los adolescentes y ofrezcan un programa que potencie los talentos e inhiba los comportamientos menos adaptativos.

Dentro de los retos educativos actuales, aún quedan muchos aspectos que requieren de la investigación rigurosa y de su aplicación en la facilitación del proceso de enseñanza-aprendizaje, favoreciendo el diseño curricular de un modelo pedagógico ajustado a la realidad neuropsicológica y educativa. Como hemos reflejado en líneas precedentes, queda un largo, a la vez que necesario, camino por explorar en el terreno de la integración entre la promoción de aspectos educativos, tanto cognitivos como motivacionales y emocionales, y la mejora de aspectos neuropsicológicos, como la funcionalidad visual o los procesos superiores implicados en el procesamiento de la información, con el fin de promover un óptimo rendimiento integral en nuestros alumnos.

\section{Limitaciones y prospectiva}

En cuanto a las limitaciones encontradas en la realización del presente estudio, una de ellas ha sido el reducido tamaño de la muestra, puesto que se considera necesario seleccionar un mayor número de sujetos y que procedan de diferentes contextos edu- cativos para obtener resultados generalizables a una población mayor. Respecto a las pruebas de evaluación aplicadas, existe un sesgo notable de la escala ACRA ya que evalúa la conducta estratégica de los alumnos sin referencia a la percepción que estos tienen sobre la relación entre lo que hacen y las metas hacia las que se encaminan, lo que constituye un olvido evidente de la dimensión intencional y metacognitiva esencial del pensamiento estratégico (Bernard, 1999). Además, tanto la STAIC y la ACRA se presentan en formato autoinforme por lo que las valoraciones son afirmaciones de los propios alumnos.

A nivel del análisis de datos y la interpretación de los resultados, tanto el reducido tamaño de la muestra que comentábamos como ciertas limitaciones a nivel metodológico y de análisis (no llevar a cabo prueba de contraste, que los resultados hayan estado en parte mediados por la sobrestimación o el hecho de realizar análisis correlacionales), han podido derivar en que los resultados no hayan sido del todo interpretables y concluyentes.

Por tanto, con el fin de solventar las dificultades descritas, se propone como prospectiva, en primer lugar, ampliar en número y variedad la muestra, en segundo lugar, la inclusión de otras variables de estudio complementarias como el autoconcepto, el nivel de autoestima, el rendimiento obtenido en otras asignaturas, la motivación, los hábitos de estudio, etc. Y, en último lugar, la realización de análisis de datos más exhaustivos tales como análisis de regresión como sustitución de las correlaciones. Con todo ello se pretendería obtener un conocimiento más amplio y consistente para poder diseñar programas de intervención neuropsicológicos que permitan trabajar de manera integrada la funcionalidad visual, las estrategias de aprendizaje y el nivel de ansiedad ante los estudios de alumnos de ESO, basado en las intervenciones desarrolladas en el campo de la Neuropsicología y la Educación en los últimos tiempos (Martín-lobo, 2015).

\section{Referencias bibliográficas}

Ballén, J. B. (2016). Relación entre la funcionalidad visual, la funcionalidad auditiva y la atención para el aprendizaje (Trabajo Fin de Máster). Universidad Internacional de la Rioja (UNIR), La Rioja.

Béjar, M. (2014). Neuroeducación. Padres y Maestros, Publicación de la Facultad de Ciencias Humanas y Sociales, 355, 49-53.

Beltrán, J., Pérez, L. F. y Ortega, M. L. (2006). CEA. Cuestionario de estrategias de aprendizaje. Madrid: TEA.

Bentosela, M. y Mustaca, A. E. (2003). El papel de la corteza prefrontal en la motivación y en la conducta intencional. Suma Psicológica, 10(2), 153-166.

Bernard, J. A. (1999). Estrategias de Aprendizaje. Madrid: Bruño.

Bolívar, J. M. y Rojas, F. (2015). Estudio de la autopercepción y los estilos de aprendizaje como factores asociados al rendimiento académico en estudiantes universitarios. Revista de Educación a Distancia, 1(44), 1-13.

Calvo, M. G. y Meseguer, E. (2002). Eye movements and processing stages in Reading. Relative contribution of visual, lexical and contextual factors. The Spanish Journal of Psychology, 5(1), 66-77.

Calvo, M. y Cano-Vindel, A. (1997). The nature of trait anxiety: Cognitive and biological vulnerability. European Psychologist, 4, 301-312.

Camarero, F., Martín, F. y Herrero, J. (2000). Estilos y estrategias de aprendizaje en estudiantes universitarios. Psicothema, 12(4), 615-622.

Castro-Sierra, E., León, F. C. P., Domínguez, L. F. G. y Rivera, A. P. (2007). Neurotransmisores del sistema límbico. Hipocampo. GABA y memoria. Segunda parte. Salud Mental, 5, 47-54. 
Cattell, R. B. y Scheier, I. H. (1961). The Meaning and Measurement of Neuroticism and Anxiety. New York: Ronald Press.

Cid, C. (2008). El uso de estrategias de aprendizaje y su correlación con la motivación de logro en estudiantes. Revista electrónica iberoamericana sobre calidad, eficacia y cambio en educación, 6(3), 100-200. Recuperado el (30 de abril de 2014) de http:// www.redalyc.org/articulo.oa?id=55160305.

Clarke, P., MacLeod, C. y Shirazee, N. (2008). Prepared for the worst: Readiness to acquire threat bias and susceptibility to elevate trait anxiety. Emotion, 1, 47-57.

Contreras, F., Espinosa, J. A., Esquerra, G., Haikal, A., Polanía, A. y Rodríguez, A. (2005). Autoeficacia, ansiedad y rendimiento académico en adolescentes. Perspectivas en psicología, 1(2), 183-194.

De la Barrera M.L., y Donolo, D. (2009). Neurociencias y su importancia en los contextos de aprendizaje, Revista digital Universitaria, 10(4) 2-24.

Delgado, B., Aparisi, D., García-Fernández, J. M., Torregrosa, M. S., Estévez, E., Marzo, J. C. y Inglés, C. J. (2018). Academic goals and learning strategies in secondary education Spanish students with social anxiety/Metas académicas y estrategias de aprendizaje en estudiantes españoles de Educación Secundaria Obligatoria con ansiedad social. Estudios de Psicología, 39(1), 58-80.

Díaz, S. B., Gómez, A., Jiménez, C., y Martínez, M. P. (2004). Bases Optométricas para una lectura eficaz. (Trabajo Fin de Máster en Optometría y entrenamiento visual). Centro de Optometría Internacional, Madrid.

Eysenck, M. W., Santos, R., Derakshan, N. y Calvo, M. G. (2007). Anxiety and cognitive performance: Attentional control theory. Emotion, 2, 336-353.

Furlán, L. A., Sánchez, J., Heredia, D., Piemontesi, S. e Illbele, A. (2009). Estrategias de aprendizaje y ansiedad ante los exámenes en estudiantes universitarios. Pensamiento psicológico, 5(5), 117-124.

Gallardo, J. R. y Gallego, J. L. (2003). Manual de Logopedia Escolar. Madrid. Editorial Aljibe.

García-Fernández, J. M., Inglés, C. J., Martínez-Monteagudo, M. C. y Redondo, J. (2008). Evaluación y tratamiento de la ansiedad escolar en la infancia y la adolescencia. Behavioral Psychology, 16, 413-437.

Gila, L., Villanueva, A. y Cabeza, R. (2009). Fisiopatología y técnica de registro de los movimientos oculares. Anales del Sistema Sanitario de Navarra, 32(3), 9-26.

González, C., Guevara, Y., Jiménez, D. y Alcázar, R. J. (2018). Relationship between Assertiveness, Academic Performance and Anxiety in a Sample of Mexican Students in Secondary Education. Acta Colombiana de Psicología, 21(1), 116-138.

Hackfort, D. y Schwenkmezger, N. (1993). Anxiety. En R. Singer, (Ed.), Handbook of research on sport psychology (pp. 328-364). New York: Macmillan Publishing.

Hasler, G., Drevets, W. C., Manji, H. K. y Charney, D. S. (2004). Discovering endophenotypes for major depression. Neuropsychopharmacology, 29, 1765-1781.

Hernández-Pozo, M. R., Coronado, O., Araújo, V. y Cerezo, S. (2008). Desempeño académico de universitarios en relación con ansiedad escolar y auto-evaluación. Acta Colombiana de Psicología 11(1), 13-23.

Herrera, L. F. (2007). Algunas consideraciones acerca de las bases neuropsicológicas de las estrategias de aprendizaje. Revista Pedagogía Universitaria, 12(2), 98-107.

Jiménez, M. A. y Huidobro, A. (2003). Cómo funciona mi cerebro. Madrid: Acento.

King, A. T. y Devick, S. (1976). Test de valoración de los movimientos sacádicos de King-Devick. Material no publicado.
Kholer, J. L. (2013). Rendimiento académico, habilidades intelectuales y estrategias de aprendizaje en universitarios de Lima. Liberabit, 19 (2), 277-288.

Lacámara, J. L. (2016). Relación entre eficacia en los movimientos sacádicos y proceso lector en estudiantes de currículo específico en educación secundaria. Alicante: Editorial Área de Innovación y Desarrollo. Doi: http://dx.doi.org/10.17993/DideInnEdu.2016.10.

López, M. (2011). Memoria de trabajo y aprendizaje: Aportes de la neuropsicología. Cuaderno de Neuropsicología, 5(1), 25-47.

López-Higes, R., Rubio, S., Villoria, C. y Mayoral, J. A. (2001). Exploración cognitiva de la lectura I: Presentación de un nuevo instrumento. Revista de Psicología General y Aplicada, 54(3), 457-495.

Lorenzo, J. R. (2002). Procesos cognitivos básicos relacionados con la lectura. Tercera parte: Procesos visuo-espaciales. Interdisciplinaria, 19(1), 1-19.

Martín-Antón, L. J., Marugán, M., Catalina, J. J. y Carbonero, M. A. (2013). Estrategias de aprendizaje de elaboración. Entrenamiento y programas. Aula Abierta, 41(1), 49-62.

Martín-Lobo, P. (2015). Procesos y programas de neuropsicología educativa. Madrid: Ministerio de Educación, Cultura y Deporte: Secretaría General Técnica: Subdirección General de Documentación y Publicaciones. Disponible en: https://sede. educacion.gob.es/publiventa/procesos-y-programas-de-neuropsicologia-educativa/ensenanza-neurologia/20612

Martín-Lobo, P., Martínez-Álvarez, I., Muelas, Á., Pradas, S. y Magreñán, A. (2018). A study of 16 years old student learning strategies from a neuropsychological perspective: An intervention proposal. Trends in Neuroscience and Education, 11, 1-8. https: //doi.org/10.1016/j.tine.2018.03.001

Martínez, I. (2015). Procesos y Programas neuropsicológicos para la lectura. En P. Martín Lobo. Procesos y programas de neuropsicología educativa (pp. 114-122). Madrid: Ministerio de Educación, Cultura y Deporte: Secretaría General Técnica: Subdirección General de Documentación y Publicaciones. Disponible en: https://sede.educacion.gob.es/publiventa/ procesos-y-programas-de-neuropsicologia-educativa/ensenanza-neurologia/20612

Medrano, S. M. (2011). Influencia del sistema visual en el aprendizaje del proceso de lectura. Revista Ciencia y Tecnología para la Salud Visual y Ocular, 9(2), 91-103.

Metsing, T. y Ferreira, J. (2008). Visual deficiencies in children from Mainstream and learning disabled schools in Johannesburg South Africa. South African Optometric, 67(4), 176-184.

Miller, D. B. y O'Callaghan, J. P. (2002). Neuroendocrine aspects of the response to stress. Metabolism, 6, 5-10.

Monereo, C. (Coord.), Castelló, M., Clariana, M., Palma, M. y Pérez, M. L. (1997). Estrategias de enseñanza y aprendizaje: Formación del profesorado y aplicación en la escuela. Barcelona: Graó.

Mora, F. (2002). ¿Cómo funciona el cerebro? Madrid: Alianza.

Muelas, A. (2015). Procesos y Programas de habilidades superiores de pensamiento y de estrategias de aprendizaje. En P. Martín Lobo. Procesos y programas de neuropsicología educativa (pp. 100-113). Madrid: Ministerio de Educación, Cultura y Deporte: Secretaría General Técnica: Subdirección General de Documentación y Publicaciones.

Northon, D. (2012). Las estrategias de aprendizaje: Una necesidad hoy en día en los ambientes educativos. Comunicación presentada en el Seminario Permanente de Ciencias Sociales. Facultad de Ciencias Sociales de Cuenca: Universidad de Castilla La Mancha.

Paulson, E. J. (2005). Viewing eye movements during reading through the lens of chaos theory: How reading is like the weather. Reading Research Quarterly, 40(3), 338-358. 
Rivas, F. (1997). El proceso de enseñanza-aprendizaje en la situación educativa. Barcelona: Ariel Planeta.

Roces, C., González-Pienda, J. A., y Álvarez, L. (2002). Procesos y estrategias cognitivas y metacognitivas. En J. A. González-Pienda, R. González Cabanach, J. C. Núñez y A. Valle, (Eds.), Manual de Psicología de la educación (pp. 95-116). Madrid: Pirámide.

Rodríguez, D. R., y Rosquete, R. G. (2018). Relación entre perfil motivacional y rendimiento académico en Educación Secundaria Obligatoria. Estudios sobre Educación, 34, 199-217.

Román, J. M. (1993). Entrenamiento en estrategias de aprendizaje: secuencias, principios y validación. En C. Monereo, (Ed.), Las estrategias de aprendizaje. Procesos, contenidos e interacción (pp. 169-194). Barcelona: Doménech.

Román, J. M. y Gallego, S. (2008). ACRA. Escalas de estrategias de aprendizaje (4⿳亠丷厂 Edición). Madrid: TEA Ediciones.

Rosselli, M. Matute, E., y Ardila, A. (2006). Predictores neuropsicológicos de la lectura en español. Revista de Neurología, 42 (4), 202-210.

Rubin, G. S., y Feely, M. (2009). The role of eye movements during reading in patients with age-related macular degeneration (AMD). Neuro-Ophthalmology 33(3), 120-126.

Santiuste, V., Martin-Lobo, P. y Ayala, C. (2006). Bases neuropsicológicas del fracaso escolar. Madrid: Furgaz.
Soto, M., Da Cuña, I., Gutiérrez, M., y González, A. (2012, noviembre). Percepción de estresores académicos y estrategias de aprendizaje en estudiantes de fisioterapia. Comunicación presentada en el I Congreso Virtual sobre Innovación Pedagógica y Praxis educativa (INNOVAGOGIA).

Spielberger, C. D., Gorsuch, R. L. y Lushene, R. E. (1970). STAI. Cuestionario de ansiedad estado-rasgo (7aㅡ ed. rev.). Madrid: TEA.

Tejero, C. M. (2006). Temores vinculados a la vida escolar en estudiantes de secundaria: Un estudio empírico. Organización y Gestión Educativa, 14, 26-29.

Tejedor, F.J., González, S.G. y García, M. (2008). Estrategias atencionales y rendimiento académico en estudiantes de secundaria. Revista Latinoamericana de Psicología, 40 (1), (123-132).

Tokuhama-Espinosa, T. N. (2008). The Scientifically Substantiated Art of Teaching: A Study in the Development of Standards in the New Academic Field of Neuroeducation (mind, Brain, and Education Science). East Eisenhower Parkway: ProQuest.

Vernett, M., Yang, Q. y Kapoula, Z. (2011). Guiding Binocular Saccades during reading: A TMS Study of the PPC. Frontiers in Human Neuroscience, 7(5), 1-8.

Zhang, H., Yan, H. M., Kendrick, K. M. y Li, C. Y. (2012). Both lexical and non-lexical characters are processed during saccadic eye movements. PLoS ONE 7(9): e46383. doi:10.1371/ journal.pone.0046383. 